\title{
FACTORS AFFECTING THE SUCCESS RATE OF RENAL STONE TREATMENT BY EXTRACORPOREAL SHOCK WAVE LITHOTRIPSY
}

\author{
HAQUE AHMA ${ }^{1}$, ISLAM MW ${ }^{2}$, KALLOL HK ${ }^{3}$, BABUL MSA ${ }^{4}$, RAHMAN MH ${ }^{5}$, CHOWDHURY SA ${ }^{6}$
}

\begin{abstract}
:
ESWL is one of the treatment modalities for kidney stones smaller than $2 \mathrm{~cm}$. However, not all ESWL treatments are successful. The success rate has been reported to be between $50 \%$ to $87 \%$, depending on various factors.

Aim: This study was conducted to evaluate Factors affecting the success rate of renal stone treatment by extracorporeal shock wave lithotripsy (ESWL).

Materials and methods: The study was carried out for a period of one year where total 96 patients with single or multiple radio-opaque renal stones treated with ESWL monotherapy using Stortz Modulith SLX-F2 lithotriptor were included. The results of treatment were evaluated after 3 months of follow-up. Treatment success was defined as complete clearance of the stones or presence of clinically insignificant residual fragments $(<4 \mathrm{~mm})$. The results of treatment were correlated with the patient characteristics (age, sex, body mass index) and stone features (size, site, number \& radio density).
\end{abstract}

Results: At 3-months follow-up, the overall success rate was 76\%. Among them, repeated ESWL sessions were required in 19 patients (53.9\%). Post-ESWL complications were recorded in 8 patients (12.5\%). Four factors had statistically significant impact on the success rate, namely stone site, size (the largest diameter of the stone), stone number, BMI (body mass index) of the patient. The success rate is highest for stones located in the upper calyx $(26 / 26 ; 100 \%)$ and lowest for those located in lower calyx (15/20; 75\%) $(p=0.019)$. Stone with a largest diameter of $<15 \mathrm{~mm}$ are associated with a success rate of 93.6\% (59/63), compared to $75.82 \%$ (25/33) for those with a diameter of $>15 \mathrm{~mm}(p=0.01)$. The success rate is also higher for single stone (76/ 84; 90.5\%) than multiple stones (8/12; 66.7\%) ( $p=0.02)$. Patients with lower BMI $(<24)$ have a better success than higher BMI (>25) ( $p=0.001)$. Other factor including age, sex and stone radio density compared to ipsilateral 12th rib have no significant impact on the success rate.

Conclusion: The success rate for ESWL for the treatment of renal stones can be predicted by stone size, location, number, and patient BMI.

J Dhaka Med Coll. 2018; 27(1) : 72-78

\section{Introduction}

Urolithiasis is a problem that has confronted by clinicians since the time of Hippocrates and the prevalence of urolithiasis is approximately 4 to 15 percent in general population and the estimated lifetime risk of developing a kidney stone is about 12 percent for white males. Approximately 50 percent of patient with urinary calculi have a recurrence within 10 years. ${ }^{1}$

Renal stones are common approximately 50\% of patient between the ages of 30 to 50 years. The male-female ratio is $4: 3$. Calculi smaller than $0.5 \mathrm{~cm}$, pass spontaneously unless they are impacted. Any surgical intervention carries risk of complication and needless intervention

1. Dr. Abul Hasanat Muhammad Afzalul Haque, MS (Urology), Assistant Registrar, Dept. of Urology, Dhaka Medical College Hospital, Dhaka

2. Dr. Md. Waliul Islam, MS (Urology), Associate Professor, Dept. of Urology, National Institute of Kidney Diseases \& Urology, Dhaka.

3. Dr. Humayun Kabir Kallol, FCPS (surgery), Medical officer, Dept. of Urology, National Institute of Kidney Diseases \& Urology, Dhaka

4. Dr. Md. Shaful Alam Babul, Resident (urology), National Institute of Kidney Diseases \& Urology, Dhaka

5. Dr. Muhammad Habibur Rahman, Assistant Registrar, National Institute of Kidney Diseases \& Urology, Dhaka

6. Shafiqul Alam Chowdhury, Professor of Urology, Dhaka Medical College Hospital, Dhaka

Correspondence: Dr. Abul Hasanat Muhammad Afzalul Haque , MS (Urology), Assistant Registrar, Dept. of Urology, Dhaka Medical college hospital, Dhaka. Cell No.01712046432,E-mail:afzalranadmc@gmail.com

Received: 21 July 2017 Revision: 01 August 2017

DOI: $h t t p: / / d x . d o i . o r g / 10.3329 / j d m c . v 27 i 1.38950$

Accepted: 01 September 2017 
should be avoided. Small renal calculi may cause symptoms by obstructing a calyx or acting as a focus for secondary infection. However most can be safely observed until they pass. ${ }^{2}$

The development of endourological and extracorporeal lithotripsy techniques led to an increasing number of options for the management of renal calculi. Each of the methods available needs to be evaluated in term of its stone clearance rate, potential morbidity and cost effectiveness. Extracorporeal shock wave lithotripsy (ESWL) is an effective, well established method for treatment of renal calculi. ${ }^{3,4}$

Chaussy et al was the first to report the clinical application of shock wave lithotripsy in the management of kidney stones and then the management of nephrolithiasis has undergone a complete revolution. ${ }^{5}$ For most renal stone smaller than $20 \mathrm{~mm}$, ESWL is the most effective primary treatment modality. ESWL is effective in all calyceal locations which are less than $20 \mathrm{~mm}$. The success rate of ESWL has been depending on stone size, stone location, stone number, renal morphology, congenital anomalies and stone composition. ${ }^{4}$

Stone radio density, a useful parameter for predicting outcome of ESWL for stone d" $20 \mathrm{~mm}$. Mina suggests that for stones $<20 \mathrm{~mm}$ within renal pelvis, the value of radiographic appearance of a stone alone in determining treatment outcome on the doli machine is somewhat limited. ${ }^{6}$ There seems to be tendency for a worse outcome for stone 11 to $20 \mathrm{~mm}$ that have a radio density greater than 12 th rib. ${ }^{7}$

Treatment outcome after lithotripsy depends on several factors. The type of lithotriptor, stone characteristics (number, size, composition and location), patient characteristics and renal anatomy and function are important factors for determining treatment characteristics and outcome. Although the role of shock wave lithotripsy for management of lower pole nephrolithiasis has been questioned in some studies, Overall stone free rates after ESWL vary from $50 \%$ to $87 \%$, depending on many factors affecting the overall success rate. ${ }^{1,8}$ On the other hand, shock wave lithotripsy is not without complications and renal trauma from treatment in time may lead to hypertension and renal insufficiency. Factors associated with increased renal damage due to shock wave lithotripsy include high shock wave number $\&$ energy. 9

\section{Materials \& Method}

This hospital based prospective study was conducted on the patients with renal stone (d" $20 \mathrm{~mm}$ ), in OPD basis in the department of Urology, NIKDU, Dhaka from July 2015 to June 2016. All patients were evaluated by detailed history, physical examination and some investigations. Urinalysis, urine culture and sensitivity, complete blood count (CBC), blood urea nitrogen (BUN), serum Creatinine, coagulation profile and plain X-Ray KUB region, ultrasonography of KUB region, IVU or Non contrast CT Scan of KUB region were done. Patient with documented urinary tract infection were treated with appropriate antibiotic before surgery. Inclusion criteria were patients with renal stones attended at the outpatient department as well as admitted in NIKDU- who are selected for ESWL; Age e"18 years irrespective of sex and BMI; Size of stone will be $>5 \mathrm{~mm}$ or d" $20 \mathrm{~mm}$ (largest diameter of stone) irrespective of site, laterality, number (single or multiple) and stone composition. Exclusion criteria were age $<18$ years; patient with ureteric stricture, coagulopathy, nonfunctioning kidney and congenital anomalies of kidney and urinary tract; stone size $>20 \mathrm{~mm}$; recurrent stones; physical disfigurement eg. Kyphosis, Scoliosis, Lordosis; Spina bifida and spinal cord injury; pregnant women. Patients, selected for ESWL according to inclusion and exclusion criteria, underwent ESWL using the MODULITH SLXF2 (STORZ, Switzerland). All the patients were nothing per oral from morning and were given intravenous fluid with diclofenac sodium suppository 30 minutes before ESWL. In a single session, maximum of 3000 shock waves were given. Repeated sessions of ESWL were given for an incomplete fragmented calculus after 3 weeks, highest upto 3 sessions.

The patients were termed as ESWL failure when no fragmentation or incomplete fragmentation found after three sessions. Patients were evaluated for stone clearance, time to stone 
clearance, number of ESWL sessions, pain intensity, incidence of steinstrasse, and any side effects at 1, 2, and 3 months. Visual analogue scale was used to measure the pain intensity.

Treatment success was defined as a complete stone clearance or clinically presence of insignificant residual fragments (CIRFs) (Stone Size $<5 \mathrm{~mm}$ ). Failure was defined as presence of significant residual fragment (SRFs) after 3rd month.

Statistical analysis was done with the data of all 96 patients from the master data sheet. The success rate was correlated with characteristics of the patients and stone feature with chi square test by using SPSS program version 22. A p value $<0.05$ was considered statistically significant.

\section{Results}

At 3 months follow-up of 96 cases complete stone free were observed in 68 patients $(70.8 \%)$, clinically insignificant residual fragments (CIRFs) were observed in patients $16(16.7 \%) \&$ significant residual fragments (SRFs) were observed in 12 patients (12.5\%).

\section{Table-I}

Distribution of the study patients by stone clearance rate $(n=96)$

\begin{tabular}{lcc}
\hline Parameters & No. of patients & Percentage (\%) \\
\hline Success & & \\
Stone-free & 68 & 70.8 \\
CIRFs & 16 & 16.7 \\
Failure & & \\
SRFs & 12 & 12.5 \\
\hline Total & 96 & 100.0 \\
\hline
\end{tabular}

At 3 months follow-up, number of overall success were $84(87.5 \%)$ and number of failure were $12(12.5 \%)$ shown in Fig-1. Among 96 cases, 45 patients (46.9\%) needed single sessions of ESWL for success. Repeated treatment was needed in 51 patients $(53.1 \%)$. Among the re-treatment group 28 patients $(54.9 \%)$ needed two and/or three sessions of ESWL to ensure success. The mean number of shocks per patient was $4883 \pm 2382$. The mean voltage was $5.76 \pm 0.68 \mathrm{kv}$. Among the failure group 2 patients were with open surgery and rest of them were referred to an urologist for post-ESWL auxiliary procedure. Among the 96 cases, post-ESWL complications were encountered in 12 patients (12.5\%).

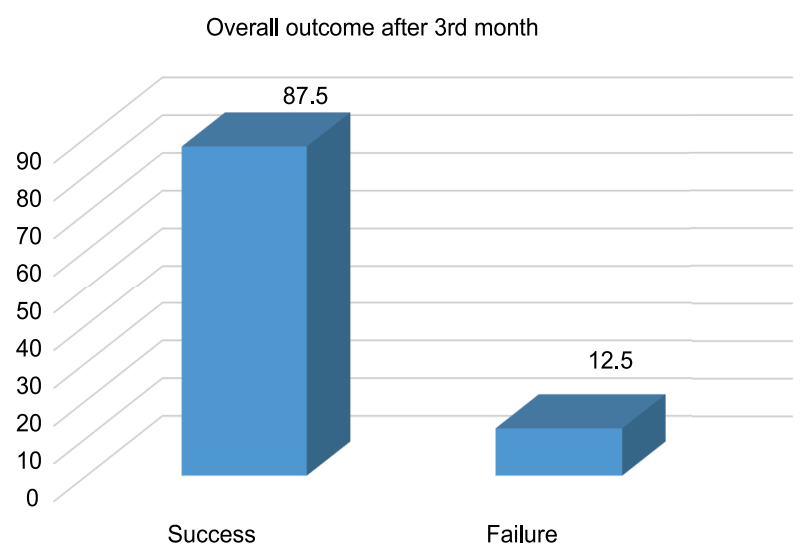

Fig-1: Bar diagram of overall success \& failure after $3^{\text {rd }}$ month. Success 86 (87.5\%), failure 12 (12.5\%).

The mean $( \pm$ SD)age of 96 patients was $38.6 \pm 10.28$ years (ranging from 19 to 60 ). The number of patients with age d" 40 years were $52(54.2 \%)$, among them number of success were $46(88.5 \%) \&$ age $>40$ years were 45 $(45.8 \%)$, among them number of success were $38(86.3 \%)$. $P$ value was $>0.05$, that was not statistically significant.

Among 96 cases, males were 53(55.2\%), among them number of success rate were $47(88.8 \%)$. The series also includes 43 females $(44.8 \%)$, among them number of success were $37(86.0 \%)$. $P$ value was $>0.05$ that was not statistically significant.

Among the 96 patients mean height of the patients was $1.56 \mathrm{~m}$ (1.56 \pm 0.073$)$. Minimum height was 1.40 meter and maximum was 1.70 meter. The mean weight of the patient was 57.45 $\mathrm{kg}(57.35+6.8)$. The minimum weight was 42 $\mathrm{kg} \&$ maximum was $70 \mathrm{~kg}$. The mean BMI of 96 patients was $23.27 \pm 1.68$ (ranging from 19.78 to 26.22). The number of patient BMI $<24$ (ranging from 19 to 24 ) were 67 (69.8\%), among them number of success were $65(97.0 \%) \&$ patient BMI >24 (ranging from 24 to 27) were $29(30.2 \%)$, among them number of success were $19(65.5 \%)$. So the success rate decreased from $97.0 \%$ to $65.5 \%$ for patient BMI $(19-24)$ to $(>24-27)$ respectively. $P$ value was $<0.001$, that was statistically significant. 
The mean stone size of 96 patients was 14.21 $\mathrm{mm}(14.21 \pm 4.61)$. The smallest stone size was $6 \mathrm{~mm} \&$ largest stone size $20 \mathrm{~mm}$. The sizes of the stones were divided into two groups. In one group the no. of stones $<15 \mathrm{~mm}$ (ranging $>4 \mathrm{~mm}$ to $15 \mathrm{~mm}$ ) were $63(65.6 \%)$, among them no. of success were $59(93.6 \%)$ and another group the no. of stones size $>15 \mathrm{~mm}$ (ranging $16 \mathrm{rnm}$ to $20 \mathrm{~mm}$ ) were $33(34.4 \%)$, among them no. of success were $25(75.8 \%)$. So in this study, the success rate for stones $<15 \mathrm{~mm}$ was $93.6 \%$, while it was $75.8 \%$ for stone $>15 \mathrm{~mm}(\mathrm{p}=0.011)$. That was statistically highly significant.

The series included the number of stones in the upper calyx were $26(27.3 \%)$, middle calyx were $28(29.2 \%)$, lower calyx were $20(20.8 \%) \&$ renal pelvis were $22(22.9 \%)$, where the number of success were $26(100 \%), 22(78.6 \%), 15(75.0 \%)$ $\& 21(95.5 \%)$ respectively. Success rate was decreased from $100 \%$ to $95.5 \%$ for upper calyx and renal pelvis, respectively. It was also decreased from $78.6 \%$ to $75.0 \%$ for stones middle calyx and lower calyx, respectively $(p=.019)$. That was statistically significant.
Patients with single stone were $84(87.5 \%)$, among them number of success were $76(90.5 \%)$. On the other hand, patients with multiple stones were $12(12.5 \%)$, among them number of success were $08(66.7 \%)(\mathrm{p}=0.020)$. That was statistically significant.

The number of stones with radiodensity $<12^{\mathrm{m}}$ rib were $56(59.3 \%)$, equal to $12^{\text {th }}$ rib were $22(22.9 \%) \&>12^{\text {ih }}$ rib were $18(18.8 \%)$, among them number of success were $52(92.9 \%)$, $18(81.8 \%) \& 17(77.8 \%)$ respectively. Here success rate was gradually increasing with decreasing the radiodensity. But $p$ value was $>0.05$ that was not statistically significant.

In 96 cases, post-ESWL complications were encountered in 12 patients (12.5\%), Among them, 6 patients (6.3) were severe pain, 4 patients $(4.2 \%)$ were massive haematuria $\&$ pain. Two patients $(2.1 \%)$ were ureteric obstruction along with haematuria and pain.

Table II

$B M I\left(\mathrm{~kg} / \mathrm{m}^{2}\right)$ and stone features in correlation with success rate $(n=96)$

\begin{tabular}{lccccc}
\hline P-value & BMI $\left(\mathrm{kg} / \mathrm{m}^{2}\right)$ & No. of $\mathrm{pts}$ & $\%$ & No. of successRate & \% success rate \\
\hline$<0.001$ & $<19(19.0-24.0)$ & 67 & 69.8 & 65 & 97.0 \\
& $24(24.01-27)$ & 29 & 30.2 & 19 & 65.5 \\
\hline
\end{tabular}

Table III

Stone size and stone features in correlation with success rate ( $n=96)$

\begin{tabular}{lccccc}
\hline P-value & Stone size & No. of pts & $\%$ & No. of successRate & \% success rate \\
\hline $0.011^{*}$ & $\leq 15 \mathrm{~mm} \mathrm{(>4-15)}$ & 63 & 65.6 & 59 & 93.6 \\
& $>15 \mathrm{~mm}(16-20)$ & 33 & 34.4 & 25 & 75.8 \\
\hline
\end{tabular}

Table IV

Stone site and stone features in correlation with success rate ( $n=96)$

\begin{tabular}{llcccc}
\hline P-value & Stone site & No. of pts & $\%$ & No. of successRate & \% success rate \\
\hline 0.019 & Upper calyx & 26 & 27.3 & 26 & 100.0 \\
& Middle calyx & 28 & 29.2 & 22 & 78.6 \\
& Lower calyx & 20 & 20.8 & 15 & 75.0 \\
& Renal pelvis & 22 & 22.9 & 21 & 95.5 \\
\hline
\end{tabular}


Table V

Distribution of the study patients by post ESWL complication ( $n=96)$

\begin{tabular}{lcc}
\hline Complications & $\begin{array}{c}\text { No. of } \\
\text { patients }\end{array}$ & $\begin{array}{c}\text { Percentages } \\
(\%)\end{array}$ \\
\hline $\begin{array}{l}\text { Severe pain } \\
\text { Massive haematuria }\end{array}$ & 6 & 6.3 \\
$\begin{array}{l}\text { \& severe pain } \\
\text { Ureteric obstruction, }\end{array}$ & 2 & 4.2 \\
$\begin{array}{l}\text { massive } \\
\text { No complications }\end{array}$ & 84 & 2.1 \\
Total & 96 & 100.0 \\
\hline
\end{tabular}

All the complications were managed conservatively according to standard protocol. No complications were encountered in 84 patients $(87.5 \%)$.

\section{Discussion:}

At 3-months follow-up, the overall success rate was $87.5 \%$. This result was matching with some similar previous studies that reported stone free rates were $75-85 \%$ for treatment of renal stones by ESWL. 1, 4, 10 This study examined only four factors that had a significant impact on the success rate namely stone size, site, number of stone \& BMI of the patient. Other factors like age, sex $\&$ radiodensity had no significant impact on the success rate.

In this study, stone size was a significant predictor of ESWL outcome. The success rate for stones $<15 \mathrm{~mm}$ was $93.6 \%$, while it was $75.8 \%$ for stone for $>15 \mathrm{~mm}$ ( $\mathrm{p}=0.011)$. AI-Ansari et al. did a prospective study under 427 patients with single or multiple stones $(<30 \mathrm{~mm})$ underwent ESWL monotherapy using SL20 lithotriptor. ${ }^{4}$ At 3-months follow-up, the overall success rate was $78 \%$. There 10 prognostic factors were studied, 5 had $a$ significant impact on the success rate namely renal morphology, congenital anomalies, stone size, stone site and number stone treated stones, other factors including age, sex, nationality, stone nature and ureteric stenting had no significant impact on the success rate.

In this study, as in others stone size had a significant predictive impact as factor of ESWL outcome. ${ }^{3,4,5,11}$ In another study, Lalak et al. evaluated the outcome of ESWL of 500 renal calculi using the dornier compact delta lithotripter. ${ }^{10}$ Here the authors found the overall stone free rate was $66 \%$, while $<10 \mathrm{~mm}$ in size was $76 \%$ at 6 months follow-up. For $10-$ $20 \mathrm{~mm}$ stones, the success rate was $66 \%$, while the rate for stones $>20 \mathrm{~mm}$ in size was $47 \%$. Here the authors did not recommend ESWL as primary therapy for stones $>20 \mathrm{~mm}$ in size. ${ }^{10}$

In the present study, the success for stones located in the renal pelvis, upper, middle and lower calyces were $95.50 \%, 100 \%, 78.6 \% \&$ $75.0 \%$ respectively $(\mathrm{p}=0.019)$. This finding was supported by similar previous studies, where for upper and lower calyceal stones free rate ranges from $90 \%$ to $70 \%$ respectively, whereas that for lower calyceal and multiple site stones ranges from $70 \%$ to $50 \%$ respectively. All the studies had shown that better stone clearance rate were in the renal pelvis, upper, $\&$ middle calyx than stone in lower calyx. $1,4,8,12$

In this study, the success rate for stones located in the lower calyx was $75 \%$. This result is in agreement with a study done by Chen who evaluated the impact of radiological anatomy as predictive factors of lower calyceal stone after ESWL. ${ }^{13}$ Here 112 patients with a solitary lower calyceal stone measuring $20 \mathrm{~mm}$ or less in size were enrolled in that retrospective study. Pretreatment IVU was reviewed for measuring the anatomical predictors, such as lower pole infundibular length (IL), infundibular width (IW) and infundibulopelvic angle (IPA), while the stone location and size were determined on plain abdominal X-ray. All treated with Siemens Lithostar Plus lithotriptor and were followedup for 3-months. Three months after ESWL, only $49(43.7 \%)$ patients were stone free. Under multivariate analysis with logistic regression, smaller stone size $(10 \mathrm{~mm}$ or less, $\mathrm{p}=0.005)$ and greater IW $(4 \mathrm{~mm}$ or more, 0.029) were significant favorable predictors for better stone clearance. The authors concluded, in addition to the influence of stone size, lower pole anatomy especially IW, had a significant impact on stone clearance for lower calyceal stone after ESWL, that was similar with other studies. ${ }^{14,} 15$

In the present study, stone number had a significant impact on stone clearance by ESWL. The success rate for single stone was $90.5 \&$ 
$66.7 \%$ for multiple stones. This result is similar to that of Abdel Khalek et al., where the authors did a studied 2954 patients with single or multiple radiopaque renal stones $(<30 \mathrm{~mm})$ underwent ESWL monotherapy. The results of treatment were evaluated after 3 months of follow up. By a multivariate regression model analysis the authors found that success rate was lower in multiple renal stones than single stone. ${ }^{1}$

In the present study, stone radiodensity alone was not a useful parameter for outcome of Extracorporeal Shock Wave Lithotripsy. This finding was supported by Mina et al. The authors studied 211 patients with solitary renal pelvic stones $<2 \mathrm{~cm}$ by Dornier Doli 50 Lithotriptor. The radiodensity was compared to ipsilateral $12^{\text {th }}$ rib. Following after 3 months follow-up they declared that there was no corelation between stone radiodensity and stone composition. For stone $<10 \mathrm{~mm}$ within renal pelvis, the SFRs were similar (71-74\%) regardless of stone radiodensity. For stone between 11 to $20 \mathrm{~mm}$, the SFR was $60 \%$, if the stone had a radiodensity $>12^{\text {th }}$ rib compared to a SFR of $71 \%$, if the stone radiodensity was $<12^{\text {th }}$ rib. However, these differences in SRFs were not statistically significant. ${ }^{6}$ In this study, we also had shown that, success rate was gradually decreasing with increasing the radiodensity of stone, but it was not statistically significant $(\mathrm{p}=0.128)$.

In the present study, success rate was significantly higher $(86 \%)$ in patients with BMI 19 to 24 compared to BMI 24 to 27 (57\%). This result was also matching with Ackermann et al. who stated that BMI influences the outcome of ESWL. They found that body mass index (BMI) and stone number were the only significant predictors. The authors stated that the best chance of success for ESWL was found in patients with BMI 20 to $28 .{ }^{16}$ But Robert et al. found patients with a BMI $>25$ had a worse outcome after ESWL that matched with present study. ${ }^{17}$

\section{Conclusion}

The overall success rate of ESWL using Stortz Modulith SLX-F2 Lithotriptor for treatment of renal stones was $87.5 \%$. The success rate gradually decreased in relation to increasing the size of the stone. But it was higher in the upper calyx pelvis and middle calyx than in the lower calyx and multiple sites of kidney. Success rate was higher for patient BMI <24. Repeated sessions were needed in $53.1 \%$ and overall complication rate was $12.5 \%$. Factors that significantly affected the success rate included stone size, stone location, multiple stones and patients BMI.

\section{References}

1. Abdel-Khalek M, Sheir KZ, Mokhtar AA, Eraky I, Kenawy M, Bazeed M. Prediction of success rate after extracorporeal shock-wave lithotripsy of renal stonesa multivariate analysis model. Scand J Urol Nephrol. 2004; 38: 161-7.

2. Fowler CG, The kidney and ureters. In: Williams MS, Buiscrode CJK. (Eds). Bailey and Love's Short Practice of Surgery, $25^{\text {th }}$ edition, Hodder Arnold publishers Ltd. London. 2008; P. 1296-1297

3. Cohen TD, Preminger GH. Management of calyceal calculi. J Urol Clin North Am, 1997; 24: 81-86.

4. Al-Ansari A, As-Sadiq K, Al-Said S, Younis N, Jaleel OA, Shokeir AA. Prognostic factors of success of extracorporeal shock wave lithotripsy (ESWL) in the treatment of renal stones. Int Urol Nephrol. 2006; 38: $63-7$.

5. Chaussy C, Brendel W, Schmied E. Extra corporeally induced destruction of kidney stones by shock waves. J Lancet.1980; 2: 1265-1268.

6. Mina SK, Paul GF, Noei S, Paramijit SC. Is stone radiodensity a useful parameter or predicting outcome of Extracorporeal Shockwave Lithotripsy for stones <2cm?. Int Braz J Urol. 2005; 31(1): 3-9.

7. Tuckey J, Devasia A, Murtha L, Ramsden P, Thomas D. Is there a simpler method for predicting lower pole stone clearance after shock wave lithotripsy than measuring infundibulo-pelvic angle?. J Endourol. 2000; 14: 475-8.

8. Coz F, Orvieto M, Bustos M, Lyng R, Stein C, Hinrich A, San I. Extracorporeal shock wave lithotripsy of 2000 urinary calculi with the modulith SL-20; success and failure according to size and location of stones. Journal of Endourology. 2009; 14(3): 239246

9. Willis LR, Evan AP, Lingemann JE. The impact of high dose lithotripsy on renal function. J Contemp Urol. 1999; 11: 45.

10. Lalak NJ, Moussa SA, Smith G, Toiley DA. The Dornier Compact Delta lithotripter: the first 500 renal calculi. J Endourol, 2002; 16: 3-7. 
11. Krings F, Tuerk CH, Steinkogie I, Marfcerger M. Extracorporeal Shock Wave Lithotripsy ("stirrup") promotes discharge of persistent calyceal stone fragments after primary extracorporeal shock wave lithotripsy. J Urol, 1992; 1440-2.

12. Sorensen CM, Chandhoke PS. Is lower pole calyceal anatomy predictive of ESWL success for primary lower pole kidney stones?. J Urol. 2002; 168: 2377 2382 .

13. Chen R, Streem SB. Extracorporeal shock wave lithotripsy for lower pole calculi; long-term radiographic and clinical outcome. J Urol. 1996; 156: 1572-1575.

14. Elbahnasy AM, Shalhav AL, Hoeig DM, Elashry OM, Smith DS, Me Dougall EM et al. Lower caliceal stone clearance after shock wave lithotripsy. J Urol. 2008; 163: 7-8.

15. Lin CC, Hsu YS, Chen KK. Predictive factors of lower calyceal stone clearance after extracorporeal shock wave lithotripsy (ESWL): The impact of Radiological Anatomy. J Chin Med Assoc. 2008; 71(10): 496-501.

16. Ackermann DK, Fuhrimann R, Pfluger D, Studer UE, Zingg EJ. Prognosis after extracorporeal shock lithotripsy of radiopaque renal calculi: a multivariate analysis. J Eur Urol. 1994; 25: 105-109.

17. Robert M, A'Ch S, Lanfrey P, Guiter J, Navratil H. Piezoelectric shock wave lithotripsy for urinary calculi: comparative study of stone depth in kidney and ureter treatments. J Endourol. 1999; 13: 699703. 